\title{
Leishmania infantum and Leishmania braziliensis: Differences and Similarities to Evade the Innate Immune System
}

\author{
Sarah de Athayde Couto Falcão',2, Tatiana M. G. Jaramillo', Luciana G. Ferreira², \\ Daniela M. Bernardes ${ }^{1}$, Jaime M. Santana ${ }^{1}$ and Cecilia B. F. Favali, ${ }^{1,2 *}$
}

${ }^{1}$ Department of Cell Biology, Biology Institute, University of Brasilia, Brasilia, Brazil, ${ }^{2}$ Núcleo de Medicina Tropical (NMT), University of Brasilia, Brasilia, Brazil

Visceral leishmaniasis is a severe form of the disease, caused by Leishmania infantum in the New World. Patients present an anergic immune response that favors parasite establishment and spreading through tissues like bone marrow and liver. On the other hand, Leishmania braziliensis causes localized cutaneous lesions, which can be self-healing in some individuals. Interactions between host and parasite are essential to understand disease pathogenesis and progression. In this context, dendritic cells (DCs) act as essential bridges that connect innate and adaptive immune responses. In this way, the aim of this study was to compare the effects of these two Leishmania species, in some aspects of human DCs' biology for better understanding of the evasion mechanisms of Leishmania from host innate immune response. To do so, DCs were obtained from monocytes from whole peripheral blood of healthy volunteer donors and from those infected with $L$. infantum or $L$. braziliensis for $24 \mathrm{~h}$. We observed similar rates of infection (around 40\%) as well as parasite burden for both Leishmania species. Concerning surface molecules, we observed that both parasites induced CD86 expression when DCs were infected for $24 \mathrm{~h}$. On the other hand, we detected a lower surface expression of CD209 in the presence of both L. braziliensis and L. infantum, but only the last one promoted the survival of DCs after $24 \mathrm{~h}$. Therefore, DCs infected by both Leishmania species showed a higher expression of CD86 and a decrease of CD209 expression, suggesting that both enter DCs through CD209 molecule. However, only $L$. infantum had the ability to inhibit DC apoptotic death, as an evasion mechanism that enables its spreading to organs like bone marrow and liver. Lastly, L. braziliensis was more silent parasite, once it did not inhibit DC apoptosis in our in vitro model.

Keywords: apoptosis, dendritic cells, Leishmania infantum, Leishmania braziliensis, CD209

\section{INTRODUCTION}

Leishmaniasis is a vector-borne disease that commits millions of people around the world. In 2013, around 215,000 new cases were reported in the world. Of these, around 21,000 were reported in Brazil (1). Around 0.2-0.4 and 0.7-1.2 million visceral leishmaniasis (VL) and cutaneous leishmaniasis (CL), respectively, occur each year. Over $90 \%$ of new cases of VL occur in six countries all 
over the world, including Brazil. About $95 \%$ of CL cases occur in the Americas, the Mediterranean basin, and the Middle East and Central Asia (2).

In the sandfly, the flagellated motile forms of Leishmania spp. called promastigotes progress through several morphological stages of differentiation, regulating the vector midgut environment (3). Finally, it becomes the non-dividing, infectious metacyclic promastigotes that are transmitted during a sandfly bite, when they are able to infect or be phagocyted by professional phagocytes as macrophages (4) and dendritic cells (DCs) (5). The parasite inside host cells becomes amastigote, a stage without an externalized flagellum that is capable of multiplication in antigen-presenting cells (6). CL is the most common clinical form of leishmaniasis and causes localized skin lesions, especially in arms and legs. In the New World, Leishmania braziliensis is able to cause from localized self-limited lesions to tissue destructive mucosal forms (7) that can worsen with age (8). On the other hand, visceral forms are caused by Leishmania infantum (9), and the disease, characterized by fever, weight loss, enlargement of the spleen and liver, and anemia, is fatal if left untreated (10).

Host-parasite interactions during innate immune responses determine the fate of adaptive immunity, contributing to healing or parasite persistence in leishmaniasis (11). DCs are professional antigen-presenting cells that interact with pathogens in peripheral tissues and stimulate $\mathrm{T}$ lymphocytes after migration to secondary lymphoid organs (12). In the periphery, DCs are in an immature state, with high potential to perform phagocytosis through many receptors that recognize pathogen-associated molecular patterns, as DC-SIGN (CD209) (13-15). Toll-like receptors (TLRs) are also involved in innate response to Leishmania parasites. During murine Leishmania major infection, data in literature showed that TLR9 was required for the induction of IL-12 in bone marrow-derived DCs (BMDCs) by intact L. major parasites or L. major DNA. This IL-12 production was essential for early interferon-gamma expression and NK cell activation (16). After pathogen internalization, DC is able to migrate from periphery to secondary tissues, rising MHC expression as well as other costimulatory molecules CD86 and CD83. After all these events, DCs are able to properly perform antigen presentation to T cells and determinate the fate of adaptive immune response (17). DCs can also become tolerogenic cells, as observed in the physiologic regulation of apoptosis (18). When L. braziliensis were in contact with murine CD $11 c^{+}$DCs, they were able to produce high levels of IL-12p70 as well as stimulate a significant expression of CD40 and CD83 in the surface of these DCs (19). Another study from the literature, working with mice BMDCs, showed that L. infantum could infect and survive inside these cells. Besides, they observed that L. infantum promastigotes were not able to upregulate CD40 and CD86 surface expression. The authors also observed that L. infantum was able to induce some level of IL-12p40 and IL-10, with no differences in TNF- $\alpha$ levels (20). Another group showed that bystander BMDCs from Balb/c mice increased IL-12p40 and expressed more CD40, CD86, and MHC class II in the cell surface than infected or not exposed cells. These bystander DCs induced a protective $\mathrm{CD}^{+}$IFN- $\gamma \mathrm{T}$ cells response, while $L$. infantuminfected DCs polarizing to T-bet+IFN- $\gamma+\mathrm{IL}-10+$ double producer T cells phenotype (21). On the other hand, human DCs infected with L. major showed an increase of HLA-DR, CD86, and CD40. When L. major-infected DCs were cocultured with T lymphocytes and treated with anti-CD40 ligand (CD40L), IL-12p70 and IFN- $\gamma$ production decreased, concluding that IL-12p70 and IFN- $\gamma$ production are CD40L-dependent (22). When CD86 (B7$2)^{-/-}$mice were infected with $L$. major, they presented a resistance, differently CD80 (B7-1) $)^{-/-}$, and wild-type Balb/c mice maintained its susceptibility. Moreover, CD86 ${ }^{-1-}$ produced more IL-4 than wild-type mice, suggesting that $\mathrm{CD} 86$ induced a Th2 response (23). Infection of BALB/c mice by Leishmania amazonensis led to higher accumulation of Langerhans cells, and $\mathrm{CD}^{+}$and $\mathrm{CD} 8^{+}$ $\mathrm{T}$ cells were found that produced IL-4 and IL-10. Nevertheless, L. braziliensis infection induced dermal DCs accumulation, increased effectors, and activated memory $\mathrm{CD} 4^{+} \mathrm{T}$ cells and IL-4, IL-10, IFN- $\gamma$ production by $\mathrm{CD}^{+}$and $\mathrm{CD}^{+} \mathrm{T}$ cells (24).

Many pathogens alter DC biology, favoring its persistence in the infected host. Data in literature showed that L. amazonensis is able to inhibit human DC differentiation from monocytes (25) and several intracellular signaling pathways (26), altering DC biology and function. Data from murine models of leishmaniasis show that inhibitory pathways are also involved in disease pathogenesis. One example is the OX40L-OX40 pathway that enhances Th2 responses in L. major-infected mice (27). Working with $O x 40 l^{-1-}$ mice, the authors observed that they were very susceptible to L. major and Leishmania mexicana. Interestingly, only lymphnode cells from $L$. major $O x 40 l^{-1-}$ mice produced less

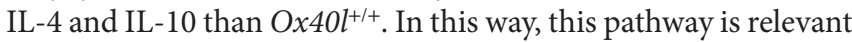
to Th2 development only in the context of $L$. major infection, but it does not alter L. major outcome of infection $(28,29)$. Other surface receptors, the programmed death ligand 1 (PD-L1) and 2 (PD-L2), were studied in susceptible mice infected with L. mexicana. PD-L1 knockout mouse demonstrated resistance, decrease of lesion size, as well as parasitic load and IL-4 production after infection. Although PD-L2 knockout mouse showed increase of lesion size, parasitic load, and antibodies production, no difference on IFN- $\gamma$ and IL-4 production was observed. Both receptors play different roles in response to L. mexicana (30).

Another way that pathogens have to adapt to the host is to alter cell death. It is well known that Mycobacterium tuberculosis induces cell death through apoptosis, but this event favors antigen presentation (31). Moreover, pathogens as Leishmania delay cell death as a way to survive inside host cell. For example, apoptosis of monocyte-derived dendritic cells (moDCs) induced by treatment with camptothecin was downregulated by infection with L. mexicana amastigotes, detected by annexin $\mathrm{V}$ binding to phosphatidylserine (32).

It is not clear how the regulation of human DC biology is affected in different ways by species of Leishmania. In this way, the aim of this study is to analyze the expression of surface molecules relevant to antigen processing and presentation as well as DCs survival after interaction with L. infantum or L. braziliensis.

\section{MATERIALS AND METHODS}

\section{Parasites}

Leishmania infantum (MHOM/BR/1974/PP75) and L. braziliensis (MHOM/BR/01/BA788) promastigotes were maintained 
in Schneider's medium (SIGMA) with $20 \%$ of calf fetal serum (GIBCO) and gentamicin $(50 \mu \mathrm{m} / \mathrm{mL})$. Leishmania stationaryphase promastigotes were quantified in Neubauer chamber. After counting, promastigotes were harvested from culture bottles, washed three times with cold PBS ( $800 \mathrm{~g}$ for $10 \mathrm{~min}$ ), and adjusted in RPMI medium to be added in DC culture.

\section{Dendritic Cell Culture}

Human DCs were differentiated from monocytes. Briefly, venous blood was collected from volunteer healthy donors $(n=8)$, and peripheral blood mononuclear cells (PBMC) were obtained by passage over a Ficoll gradient (GE Healthcare). Cells were harvested, washed, and stained with antibodies, anti-CD14, conjugated with microbeads (Miltenyi Biotec). Stained cells (monocytes) were purified after positive selection in a magnetic field. Monocytes were then counted and cultivated in RPMI 1640 medium supplemented with $2 \mathrm{mM} \mathrm{L}$-glutamine, $100 \mathrm{U} / \mathrm{mL}$ penicillin, $100 \mathrm{mg} / \mathrm{mL}$ streptomycin (GIBCO), IL-4 (800 IU/mL), and GM-CSF $(50 \mu \mathrm{g} / \mathrm{mL})$ (both from PeproTech) with $10 \%$ of fetal serum bovine (GIBCO) in 24 -wells plates $\left(5 \times 10^{5}\right.$ cells/wells) for 7 days at $37^{\circ} \mathrm{C}$ and $5 \% \mathrm{CO}_{2}$. New medium $(200 \mu \mathrm{L})$ supplemented with cytokines was added on days 3 and 6 of the culture. On day 7, DCs were infected (1 DC:10 parasites), cultivated in 24-wells plates for $24 \mathrm{~h}$ at $37^{\circ} \mathrm{C}$ and $5 \% \mathrm{CO}_{2}$, harvested, and characterized by flow cytometry (FACS Verse BD Biosciences). All experimental conditions and measurements were performed with the same donors.

\section{Flow Cytometry and Antibodies}

Dendritic cells were cocultured with Leishmania species for $24 \mathrm{~h}$, and control cells were harvested and washed with PBS. Cells were then resuspended in FACS buffer $\left(5 \times 10^{6}\right.$ cells $\left./ \mathrm{mL}\right)$ and incubated at $4^{\circ} \mathrm{C}$ for $20 \mathrm{~min}$. The cells were stained with CD1a FITC-conjugated (clone HI149), HLA-DR PE-conjugated (clone LN3), CD86 PE-Cy5-conjugated (clone IT2-2), and CD209 PE-conjugated (clone eB-h209) (all eBioscience) and incubated for $30 \mathrm{~min}$ at $4^{\circ} \mathrm{C}$. Finally, cells were washed, resuspended in FACS buffer, and acquired on a flow cytometer. For cell death experiments, after infection time, the cells were washed and resuspended in $195 \mu \mathrm{L}$ of binding buffer to each $2-5 \times 10^{5}$ cells, added $5 \mu \mathrm{L}$ of annexin $\mathrm{V}$, and incubated for $10 \mathrm{~min}$ at room temperature. The cells were washed with PBS $1 \times$, resuspended in $190 \mu \mathrm{L}$ of binding buffer, added $10 \mu \mathrm{L}$ propidium iodide (PI) (annexin $\mathrm{V}$ and propidium iodide kit, eBioscience), and acquired on a flow cytometer. Stained cells were acquired on a Verse flow cytometer (BD Bioscience) and analyzed using FlowJo Software (Tree Star, Inc.).

\section{Cytokine Assay}

Dendritic cells were infected with both Leishmania species. After $24 \mathrm{~h}$ of infection, supernatant was collected and level of TNF- $\alpha$ was measured by ELISA. TNF- $\alpha$ was quantified (test detection range from 20 to $1000 \mathrm{pg} / \mathrm{mL}$ ) with purified antihuman TNF- $\alpha$, antihuman TNF- $\alpha$ biotin, and streptavidin-alkaline phosphatase, according to the instruction of suppliers (Novex, Life Technologies, Invitrogen). Absorbance was measured and analyzed with the program SoftMax Pro (Molecular Devices).

\section{Ethical Statement}

Human blood samples were collected after the signature of an informed consent signed by all volunteers, and the project was approved by The Ethical Committee for Human Beings from the Medicine Faculty of University of Brasilia (approval no. 072/2009). Samples were collected by venous puncture by a trained and specialized laboratory technician in the Universidade de Brasília.

\section{Statistical Analysis}

The statistical analysis was conducted using GraphPad Prism Software. Samples were tested by Shapiro-Wilk normality test, and the significance of the results was calculated using parametrical paired $t$ test, and a $p$-value of $<0.05$ was considered significant.

\section{RESULTS}

\section{L. braziliensis- or L. infantum-Exposed Dendritic Cells Upregulate CD86 and Downregulate CD209 Expression}

After $24 \mathrm{~h}$, the parasite load of DCs was accessed by optic microscopy, and we observed $40.67 \pm 5.8 \%$ L. infantum (Li)-infected DCs and $42.13 \pm 3.1 \%$ L. braziliensis (Lb)-infected DCs (Figure 1A). The amastigotes number per 100 cells was $249.8 \pm 44.23$ amastigotes in L. infantum (Li)-infected DCs and $185.3 \pm 32.73$ in
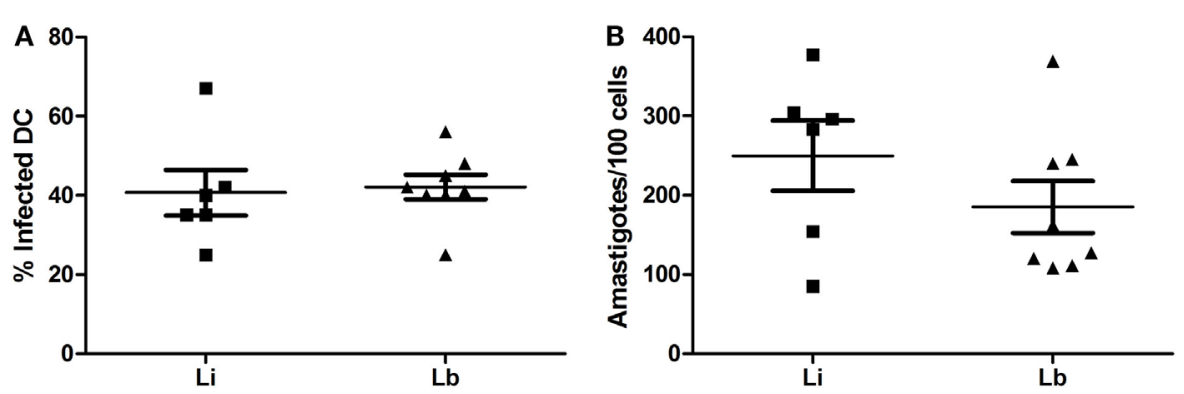

FIGURE 1 | Dendritic cells' infection by L. infantum (Li) or L. braziliensis (Lb) - immature DCs were infected in a rate of 10 Leishmania to 1 DC Infection after $24 \mathrm{~h}$, in hematoxylin-eosin (HE)-stained slides. Median \pm SD. Each point represents one donor. ${ }^{\star} p<0.01$. (A) Percentage of infected DCs. (B) Amastigotes per 100 cells. 
L. braziliensis (Lb)-infected cells (Figure 1B). Later, we decided to observe the co-stimulatory and surface molecules expression by DCs after the exposure to L. infantum or L. braziliensis. Then, we stained the cultured DCs with medium or each parasites species with anti-CD1a, anti-HLA-DR, anti-CD86, and anti-CD209 antibodies. The cells were gated by granularity and size, and the gated cells were further analyzed for expression of CD1a/ HLA-DR/CD86/CD209 surface molecules (Figure 2). Control DCs, differentiated with media only $(\mathrm{M})$, showed $56.53 \pm 8.2 \%$ of CD1a expression. This was very similar in DCs exposed to L. infantum $(60.40 \pm 8.1 \%)$ as well as exposed to L. braziliensis $(57.8 \pm 8.3 \%)$ (Figure 3A).

In the same way, we observed a consistent higher expression of HLA-DR in control cells $(73.81 \pm 5.55 \%)$-, in L. infantum $(68.27 \pm 4.8 \%)$-, and L. braziliensis $(80.89 \pm 2.7 \%)$-exposed DCs, with no significant differences between the groups (Figure 3B).

However, both Leishmania were able to induce a significant raise in CD86 expression. We observed that CD86 expression was $73.23 \pm 5.4 \%$ in control cells and increased to $78.16 \pm 4.3 \%$ in the presence of $L$. infantum and to $92.24 \pm 1.9 \%$ in the presence of L. braziliensis (Figure 3C). Moreover, this increase was significantly higher in L. braziliensis-infected DCs than in $L$. infantum-infected DCs (Figure 3C).

On the other hand, we observed that both Leishmania were able to inhibit significantly CD209 expression. Its expression was $80.09 \pm 6.8 \%$ in control cells, while in the presence of L. infantum, it was down modulated to $62.17 \pm 9.88 \%$. The presence of L. braziliensis also led to a lower CD209 expression (75.36 $\pm 7.3 \%)$ (Figure 3D). This decrease was significantly lower in L. infantuminfected DCs than L. braziliensis-infected DCs (Figure 3D).

When we analyzed the mean intensity of fluorescence (MFI) of these molecules, we observed the same effects that we observed in the percentage of expression. The MFI was similar in all experimental conditions for CD1a and HLA-DR (Figures 4A,B). On the other hand, we observed a significantly higher CD86 MFI induced by L. braziliensis $(2060 \pm 351.9)$, but no significant difference was found in the presence of $L$. infantum $(1537 \pm 169.4)$ when compared to control cells (1227 \pm 152.2) (Figure 4C). Also, as we observed in surface expression, the CD209 MFI was significantly lower in the presence of $L$. infantum $(856.1 \pm 72.8)$ or L. braziliensis $(1033 \pm 112.6)$, compared to control cells $(1127 \pm 116.7)$ (Figure 4D).
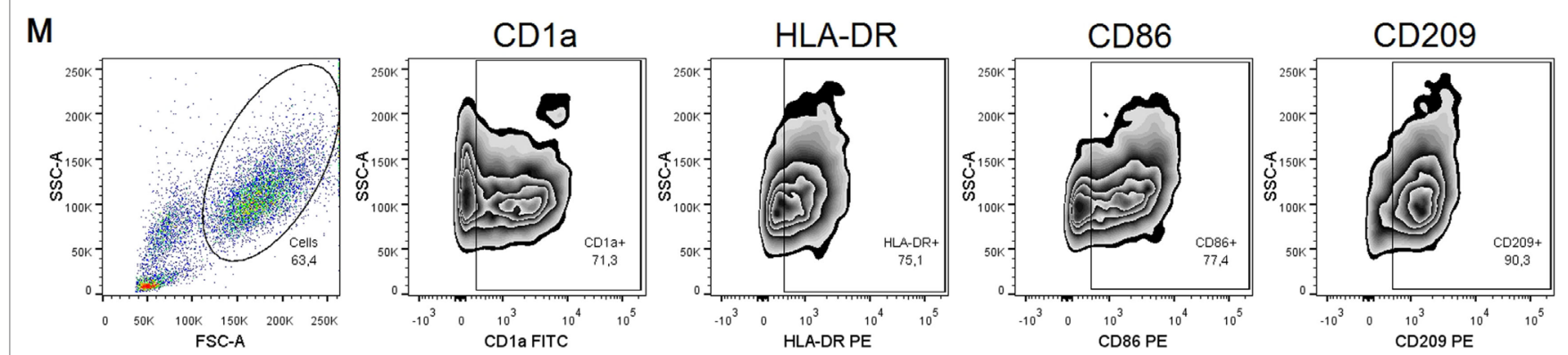

Li
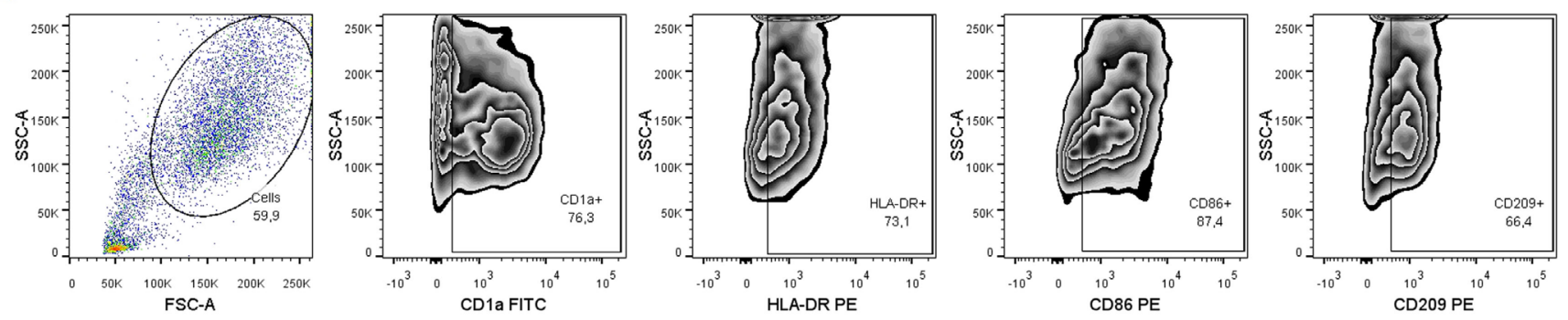

Lb
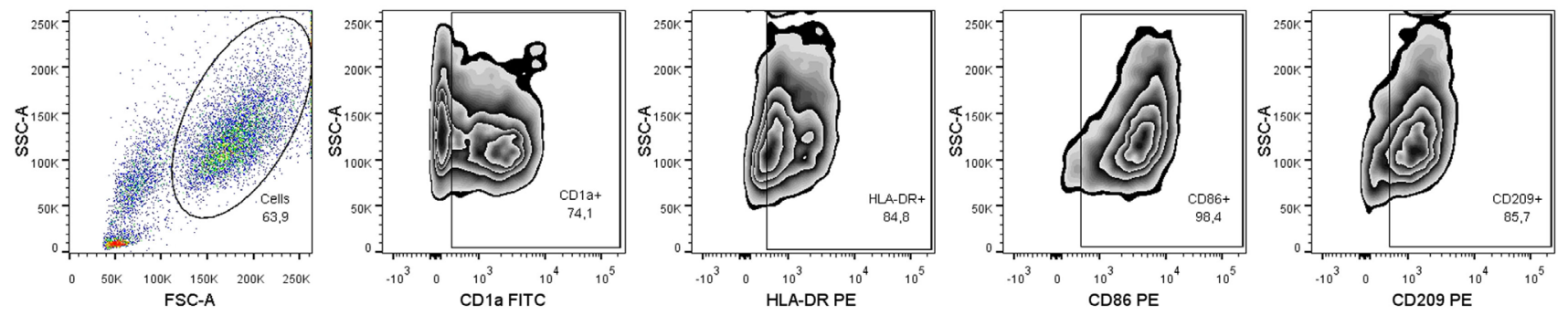

FIGURE 2 | Gating strategy to analyze DCs' regulation by Leishmania parasites - DCs in control and infected conditions were gated by granularity and size and further analyzed for expression of CD1a/HLA-DR/CD86/CD209 surface molecules. M, medium, Li, Leishmania infantum, Lb, Leishmania braziliensis. 

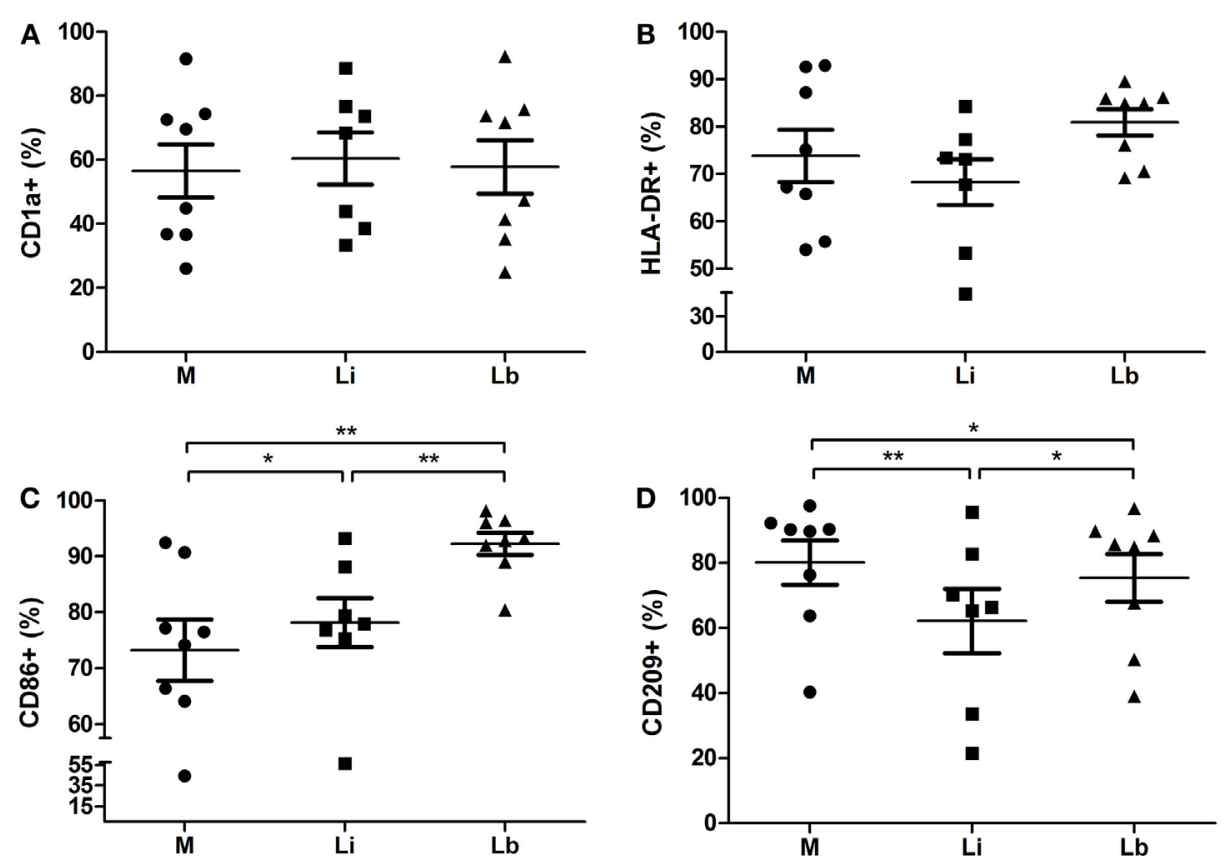

FIGURE 3 | L. braziliensis- or L. infantum-exposed dendritic cells upregulate CD86 and downregulate CD209 expression - flow cytometry analysis of surface molecule expression in immature DCs differentiated from human monocytes with cytokine-conditioned media (M) or conditioned media in the presence of viable L. infantum (Li) or L. braziliensis (Lb). CD1a (A), HLA-DR (B), CD86 (C), and CD209 (D). Median \pm SD. Each point represents one donor. ${ }^{*} p<0.05,{ }^{* *} p<0.01$.
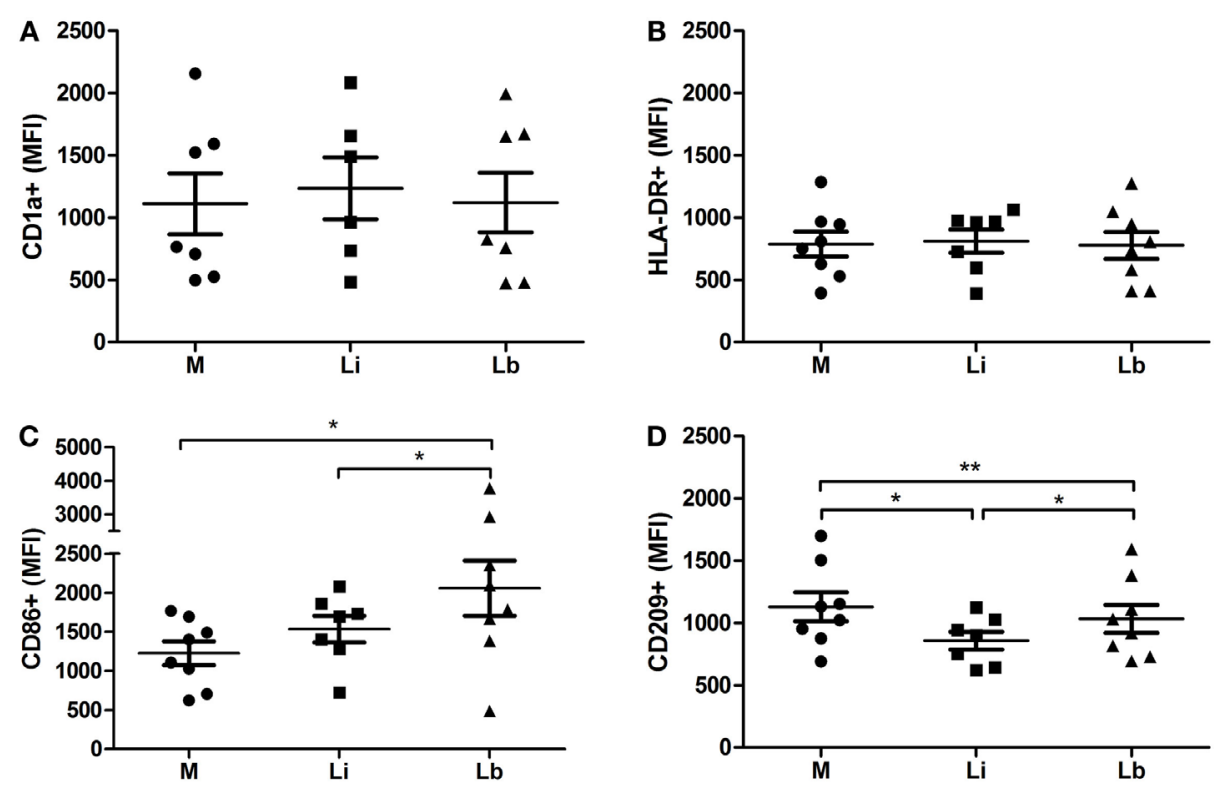

FIGURE 4 | L. braziliensis-exposed DCs increase the mean of fluorescence intensity (MFI) of CD86 and decrease CD209 - flow cytometry analyzes of the MFI in immature DCs differentiated from human monocytes with cytokine-conditioned media (M) or conditioned media in the presence of viable L. infantum (Li) or L. braziliensis (Lb). CD1a (A), HLA-DR (B), CD86 (C), and CD209 (D). Median \pm SD. Each point represents one donor. ${ }^{*} p<0.05,{ }^{* *} p<0.01$. 


\section{Exposure to L. infantum but Not L. braziliensis Decreases the Dendritic Cells' Cellular Death}

Next, we decided to evaluate if these two Leishmania species were able to induce or not cell death of DCs. To do so, after a coculture of the DCs with the two parasite species, we stained the cells with annexin V and PI (Figure 5A). We observed no significant differences between DCs only and L. braziliensis-exposed DCs in the annexin $\mathrm{V}+\mathrm{PI}-$ and annexin $\mathrm{V}+\mathrm{PI}+$ populations (Figures 5B,C, respectively). Nevertheless, the cell population that was annexin $\mathrm{V}+\mathrm{PI}-$ population significantly decreased in L. infantum-exposed DCs, from $5.7 \pm 1.0 \%$ in control cells to $2.9 \pm 0.6 \%$, that is a $50.8 \%$ decrease in DC death, showing that the L. infantum is able to inhibit cellular death of DCs (Figure 5B). However, no significant decrease was observed in the annexin $\mathrm{V}+\mathrm{PI}+$ population, $11.36 \pm 2 \%$ in control cells and $8.3 \pm 2.4 \%$ in L. infantum-exposed DCs (Figure 5C).

\section{L. infantum- and L. braziliensis-Exposed Dendritic Cells Produced TNF- $\alpha$}

Coculture with $L$. infantum or L. braziliensis triggered a significant secretion of TNF- $\alpha$ by DCs. Control cells produced $17.6 \pm 4.4 \mathrm{pg} / \mathrm{mL}$, while DCs cocultured with L. infantum induced $61.9 \pm 10.4 \mathrm{pg} / \mathrm{mL}$, a 3.5 -fold significant increase in this cytokine secretion. On the other hand, L. braziliensis stimulated significantly even more TNF- $\alpha$ secretion $(133.2 \pm 43.7 \mathrm{pg} / \mathrm{mL})$, almost an eightfold increase (Figure 6).

\section{DISCUSSION}

Many data in literature show the relevant role of DCs in diverse pathogen-host interactions, especially in the initial events of innate immunity. These initial events lead the course of adaptive response (33). For a better understanding of Leishmania and infected host regulation, we initially cocultured DCs with stationary non-opsonized L. braziliensis and L. infantum promastigotes for $24 \mathrm{~h}$. The infection rate was similar in our experiments between the two Leishmania species (around 40\%), and we found no significant difference of internalized parasites number. Concerning infection data, non-opsonized L. mexicana promastigotes presented higher phagocytosis than opsonized promastigotes or amastigotes by human moDCs after $3 \mathrm{~h}$. Six hours later, opsonized promastigotes showed higher phagocytosis than nonopsonized promastigotes and amastigotes, but no difference was observed after $24 \mathrm{~h}$ of infection (5). Infection of human DCs with L. amazonensis showed a similar rate of infection, around 45\%, and a parasite burden median of six amastigotes per DC (25).

We observed that both L. braziliensis and L. infantum were able to induce an increase in CD86 expression and also caused a decrease in CD209 (DC-SIGN) expression in human DCs. A study with $L$. amazonensis showed that the parasite modulates the co-stimulatory molecules expression during the differentiation of human moDCs, affecting the mixed leukocyte reaction and the specific T lymphocytes responses. Similar to our data, L. amazonensis also caused an increase in CD86 expression after a 24-h parasite DCs contact (25). On the other hand, murine
A
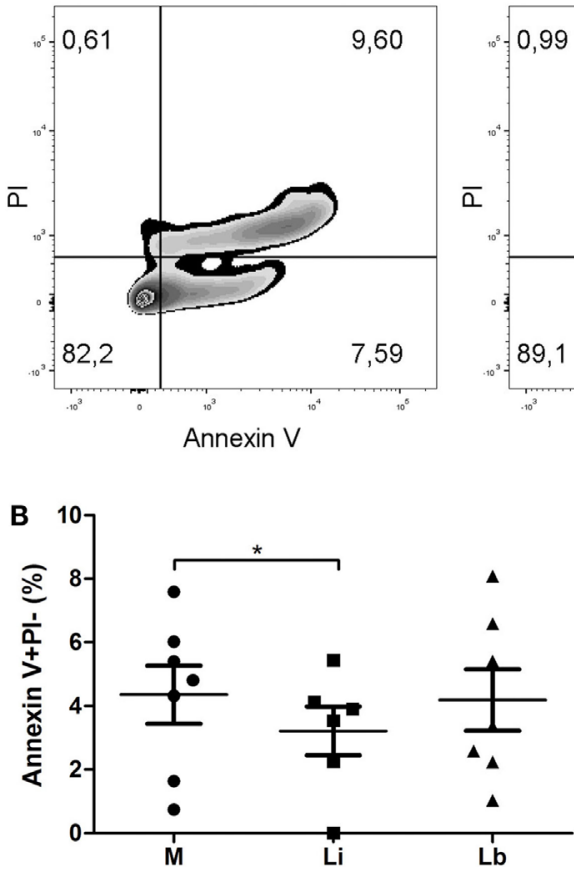

$\mathrm{Li}$
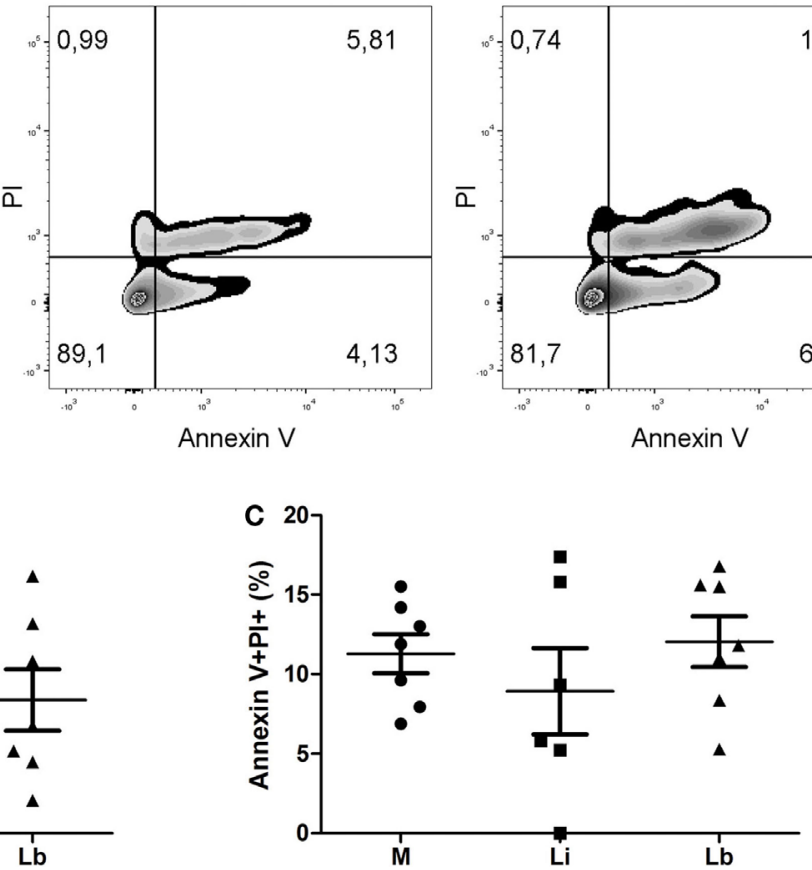

FIGURE 5 | L. infantum delayed the cellular death of dendritic cells - flow cytometry gating strategy (A) to perform analyses of annexin $\mathrm{V}$ and propidium iodide (PI) staining of control DCs (M) or treated with viable $L$. infantum (Li) or L. braziliensis (Lb). Annexin $\vee$ positive cells (B) and annexin $V$ and PI positive cells (C). Median \pm SD. Each point represents one donor. ${ }^{\star} p<0.05,{ }^{* *} p<0.01$. 


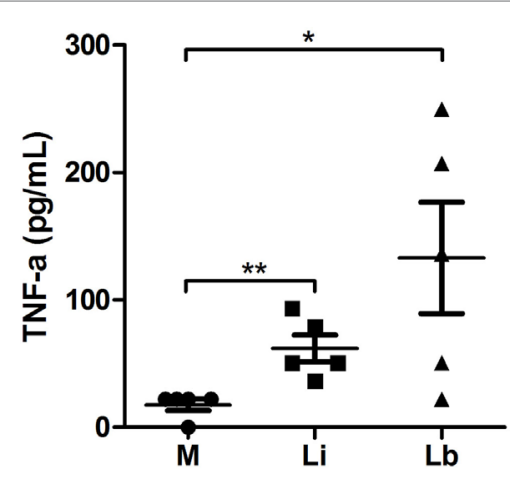

FIGURE 6 | L. braziliensis induced TNF- $\alpha$ secretion on immature DCs - cytokine levels measured on culture supernatants after $\mathbf{2 4} \mathrm{h}$ of infection. Control DCs were cultured in cytokine-conditioned media only or treated with viable $L$. infantum or $L$. braziliensis parasites. Median $\pm S D$. Each point represents one donor. ${ }^{*} p<0.05,{ }^{\star *} p<0.01$.

mature DCs infected with L. amazonensis amastigotes presented a decreased antigen presentation, CD40 and CD83 expression, and also a reduced $\mathrm{CD}^{+} \mathrm{T}$ cells priming (34). Contreras et al. (35), showed that L. mexicana infection also interfered in the DC maturation process, decreasing co-stimulatory molecules expression and antigen presentation, injuring the presentation to $\mathrm{T}$ cells by human DCs. Macrophages cultured with peripheral blood leukocytes (PBL) exhibited CD40 and CD86 expression in the absence of $L$. major, but their expression levels were higher in the presence of the parasite. After blocking CD80, CD86, both, or CD40, macrophages cultured with PBL and L. major produced less IFN- $\gamma$, IL-5, and IL-12, showing that these costimulatory pathways were important to the development of an anti-Leishmania-efficient immune response (36). On the other hand, a study performed with VL patients showed that the subset of inflammatory monocytes $\mathrm{CD} 14^{+} \mathrm{CD} 16^{+}$remained the same as in the control group. Similarly, the population of CD $54^{+} \mathrm{HLA}-\mathrm{DR}^{+}$ monocytes was significantly downregulated during active disease. Finally, they observed an increase in CD80 expression level, but a decreased CD86 expression (37). Concerning infection with L. braziliensis, data from literature showed that $\mathrm{CD} 14^{+}$monocytes of peripheral blood from CL patients had a decreased expression of CD80 and CD86 following culture with media only or after restimulation with Leishmania antigen (38). It seems that monocytes and DCs have different roles in the regulation leishmaniasis. Monocytes seem to have a role in regulating the lesion site during CL pathogenesis, and the authors mentioned before Vieira et al. (38) observed a diminished expression of B7 molecules. In our model, we worked with moDCs from healthy donors' peripheral blood. In this way, it is possible that in these initial interactions, once our experiments were performed after a 24-h infection with Leishmania parasites, these DCs regulate CD86 expression positively, being able to activate naive lymphocytes. On the other hand, the effects seem to be opposite for monocytes that migrate to lesion sites, once in patients with CL it is observed a negative regulation of CD86 (38).

Concerning CD209 expression, the decrease of this surface molecule was also observed in L. major-and L. donovani-infected human DCs (39). It is well established that the receptor CD209 can be used for internalizing microrganisms as parasites (40). In this way, our data are in accordance with data in the literature, suggesting that L. infantum and L. braziliensis attach to CD209 to access host DCs, which would explain the decrease in CD209 in the surface, as it could be internalized. Nevertheless, CD209 was involved in the phagocytosis of L. mexicana promastigotes after 3 h, but not after 6 or 24 h (5). Excreted or secreted recombinant proteins of $L$. infantum interfered in the differentiation of DCs, decreasing the HLA-DR expression but increasing the CD86 expression (41). We observed the same effect concerning CD86, but we observed no significant differences in HLA-DR surface expression caused by L. infantum or L. braziliensis in our work. Regarding CD86 and CD209 expression, we found a higher expression of CD86 in the presence of L. braziliensis than in the presence of L. infantum; however, the decrease in CD209 expression was greater in cells infected with L. infantum than in L. braziliensis-infected ones. Though, the rates of infection as well as the number of internalized amastigotes were similar between both the species of Leishmania. This can be explained by the fact that DCs have other recognition receptors that are able to recognize and be involved in Leishmania internalization, which were not investigated in this work. We can mention the mannose-fucose receptor, which is present in DCs that can recognize lipophosphoglycan (LPG), GP63, and proteophosphoglicans (PPG) present in the membrane of Leishmania species $(42,43)$. This pathway could explain the DCs' infection rate by L. braziliensis and the number of amastigotes to be similar to the L. infantum-infection. However, macrophages of B6 mice mannose receptor (MR) knockout showed an increase of parasite load after infection with L. infantum. This group showed that MR and Dectin-1 signaling pathways are essential to trigger the oxidative burst in macrophages infected with $L$. infantum, enabling parasite control (44).

Many data in literature showed the relevance of cytokines regulation in leishmaniasis pathogenesis. The presence of L. amazonensis during the differentiation of human DC inhibited IL-6 and IL-10 secretion. Besides, these DCs cocultured with $L$. amazonensis, when in contact with autologous lymphocytes, caused a lower secretion of IFN- $\gamma$ (25). Moreover, amastigotes of L. amazonensis suppressed IL- $12+\mathrm{p} 40$ and IL-10 production by murine DCs (34). However, we found a higher release of TNF- $\alpha$ after coculture of DCs and L. braziliensis or L. infantum. The excreted or secreted recombinant proteins of L. infantum also induced TNF- $\alpha$ production (41), as we saw for both Leishmania species in this work. Studies with fluorescent $L$. braziliensis parasites allowed the observation that the TNF- $\alpha$ production derived from infected DCs, but not from bystander DCs, had contact with L. braziliensis in the medium but were not infected. Moreover, soluble parasite products and the TNF- $\alpha$ released by infected DCs provided a more efficient antigen presentation by DCs (45). Data from literature evaluated TNF- $\alpha$ production from PBMC of patients and healthy individuals after stimulation with soluble L. braziliensis antigens. The results showed a range in the SD, as expected for human individuals. They showed that healthy donors produced $48.6 \pm 77.18 \mathrm{pg} / \mathrm{mL}$ of TNF- $\alpha$ (46). Another study that compared PBMC responses from patients 
with CL and mucosal leishmaniasis (ML), after stimulation with $L$. braziliensis soluble antigen, also detected a wide range of TNF- $\alpha$ levels. In CL, TNF- $\alpha$ levels ranged from 0 to $7440 \mathrm{pg} /$ $\mathrm{mL}$, while in ML patients from 892 to $9173 \mathrm{pg} / \mathrm{mL}$ (47). These differences in TNF levels are expected and may exist due to the genetic constitution of the host. On the other hand, data from literature that analyzed immune responses from patients with active VL detected some similarities among VL patients and healthy individuals, concerning cytokines regulation. Analysis ex vivo of patient's cells with active VL showed the production of high levels of anti-inflammatory cytokines and lower TNF levels, the last ones very similar to healthy controls (37). Another study with patients with active VL due to $L$. infantum also showed lower TNF- $\alpha$ levels, very similar to healthy controls (48). These observations are similar to our results in which we could not observe the individual variations in healthy donors' DCs infected with L. infantum. Another possibility is that even with similar rates of infection and parasites per cell, our results reflect the differences cited before concerning the immunogenicity of $L$. braziliensis and L. infantum.

We observed that $L$. infantum caused an inhibition of DC apoptosis. The same phenomenon was not observed for L. braziliensis. This demonstrates that possibly only L. infantum was able to stimulate the development of an environment adequate for the multiplication inside the cells and that favors a possible spread to other organs as bone marrow and liver. This surviving of DC was also seen in L. mexicana infection when apoptosis was induced by camptothecin (49). Indeed, DCs infected with L. mexicana amastigotes decreased the phosphorylation of MAP kinase p38 and JNK, causing a decreased DNA fragmentation in the camptothecin stimulated DCs. L. mexicana amastigotes activated antiapoptotic pathways, such as PI3K and AKT, allowing the inhibition of infected DCs' cell death (50). The opposite effect was observed with the bacteria Brucella abortus that induced apoptosis and necrosis of murine DCs. This apoptosis regulation was performed by caspase-2 in mouse model (51).

A very elegant paper showed that Dectin-1, MR, and DC-SIGN homolog SIGNR3 are able to recognize L. infantum, but only DC-SIGN (CD209) and MR triggered pro-IL1 beta processing

\section{REFERENCES}

1. WHO. Accelerating Work to Overcome the Global Impact of Neglected Tropical. Geneva: WHO (2012).

2. Alvar J, Vélez D, Bern C, Herrero M, Desjeux P, Cano J, et al. Leishmaniasis worldwide and global estimates of its incidence. PLoS One (2012) 7:e35671. doi:10.1371/journal.pone.0035671

3. Santos VC, Vale VF, Silva SM, Nascimento AA, Saab NA, Soares RP, et al. Host modulation by a parasite: how Leishmania infantum modifies the intestinal environment of Lutzomyia longipalpis to favor its development. PLoS One (2014) 9:e111241. doi:10.1371/journal.pone.0111241

4. Ramos PK, Brito MV, Silveira FT, Salgado CG, De Souza W, Picanço-Diniz $\mathrm{CW}$, et al. In vitro cytokines profile and ultrastructural changes of microglia and macrophages following interaction with Leishmania. Parasitology (2014) 141:1052-63. doi:10.1017/S0031182014000274

5. Argueta-Donohué J, Wilkins-Rodríguez AA, Aguirre-García M, GutiérrezKobeh L. Differential phagocytosis of Leishmania mexicana promastigotes and amastigotes by monocyte-derived dendritic cells. Microbiol Immunol (2015). 60:369-81. doi:10.1111/1348-0421.12325 in a caspase-1-dependent way, being crucial for microbicidal activity of macrophages. On the other hand, signaling through SIGNR3 seems to favor parasite survival because of its modulation of inflammasome activation (44). Once we found a possible correlation of CD209 and cell death, perhaps the internalization of L. infantum by CD209 in human DCs could also be interfering in the activation of caspase-1, causing the survival of DCs differently from the data observed on macrophages. On the other hand, L. braziliensis seems to not interfere in this pathway of cell death regulation, once we did not observe differences concerning DC survival.

In this way, we can conclude that both L. infantum and L. braziliensis possibly enter DCs through DC-SIGN, causing a positive regulation on CD86 expression as well as on TNF- $\alpha$ production after this initial interaction. Nevertheless, only L. infantum induced a survival in human DCs, probably favoring its establishment and spread in the host, differently from $L$. braziliensis that seems to be more silent in these initial events concerning DC survival. This work shed light in the differences between these two parasites and their interactions with human DCs and opens the field to study the development of intracellular signaling pathways responsible for these phenomena.

\section{AUTHOR CONTRIBUTIONS}

SF and CF: conceived and designed the experiments. SF, TJ, LF, DB, and CF: performed the experiments. SF, TJ, and CF: analyzed the data. SF, JS, and CF: contributed reagents/materials/analysis tools. SF and CF: wrote the paper.

\section{FUNDING}

SF is thankful to the CAPES and Nucleo de Medicina Tropical for providing the PNPD fellowship. CF is thankful for the Centro Nacional de Pesquisa e Tecnologia (CNPq) for providing the Grant (Edital MCT/CNPq No. 14/2011 - Universal) and Fundação de Amparo à Pesquisa do Distrito Federal (FAP-DF) for providing the Grant (Edital - Demanda Espontânea 03/2010). FINEP and Universidade de Brasilia.

6. Prina E, Abdi S, Lebastard M, Perret E, Winter N, Antoine J. Dendritic cells as host cells for the promastigote and amastigote stages of Leishmania amazonensis: the role of opsonins in parasite uptake and dendritic cell maturation. J Cell Sci (2004) 117:315-25. doi:10.1242/jcs.00860

7. Barral A, Barral-Netto M, Almeida R, de Jesus AR, Grimaldi Júnior G, Netto EM, et al. Lymphadenopathy associated with Leishmania braziliensis cutaneous infection. Am J Trop Med Hyg (1992) 47:587-92.

8. Carvalho AM, Amorim CF, Barbosa JL, Lago AS, Carvalho EM. Age modifies the immunologic response and clinical presentation of American tegumentary leishmaniasis. Am J Trop Med Hyg (2015) 92:1173-7. doi:10.4269/ ajtmh.14-0631

9. Ready PD. Epidemiology of visceral leishmaniasis. Clin Epidemiol (2014) 6:147-54. doi:10.2147/CLEP.S44267

10. Bhattacharya SK, Dash AP. Treatment of visceral leishmaniasis: options and choice. Lancet Infect Dis (2016) 16:142-3. doi:10.1016/S1473-3099(15) 00528-9

11. Gollob KJ, Viana AG, Dutra WO. Immunoregulation in human American leishmaniasis: balancing pathology and protection. Parasite Immunol (2014) 36:367-76. doi:10.1111/pim.12100 
12. Cools N, Ponsaerts P, Van Tendeloo VF, Berneman ZN. Balancing between immunity and tolerance: an interplay between dendritic cells, regulatory $\mathrm{T}$ cells, and effector T cells. J Leukoc Biol (2007) 82:1365-74. doi:10.1189/ jlb.0307166

13. Colmenares M, Puig-Kröger A, Pello OM, Corbí AL, Rivas L. Dendritic cell (DC)-specific intercellular adhesion molecule 3 (ICAM-3)-grabbing nonintegrin (DC-SIGN, CD209), a C-type surface lectin in human DCs, is a receptor for Leishmania amastigotes. J Biol Chem (2002) 277:36766-9. doi:10.1074/jbc. M205270200

14. Vannberg FO, Chapman SJ, Khor CC, Tosh K, Floyd S, Jackson-Sillah D, et al. CD209 genetic polymorphism and tuberculosis disease. PLoS One (2008) 3:e1388. doi:10.1371/journal.pone.0001388

15. Jin W, Li C, Du T, Hu K, Huang X, Hu Q. DC-SIGN plays a stronger role than DCIR in mediating HIV-1 capture and transfer. Virology (2014) 458459:83-92. doi:10.1016/j.virol.2014.04.016

16. Liese J, Schleicher U, Bogdan C. TLR9 signaling is essential for the innate NK cell response in murine cutaneous leishmaniasis. Eur J Immunol (2007) 37:3424-34. doi:10.1002/eji.200737182

17. Dalod M, Chelbi R, Malissen B, Lawrence T. Dendritic cell maturation: functional specialization through signaling specificity and transcriptional programming. EMBO J (2014) 33:1104-16. doi:10.1002/embj.201488027

18. Steinman RM, Turley S, Mellman I, Inaba K. The induction of tolerance by dendritic cells that have captured apoptotic cells. J Exp Med (2000) 191:411-6. doi:10.1084/jem.191.3.411

19. Vargas-Inchaustegui DA, Xin L, Soong L. Leishmania braziliensis infection induces dendritic cell activation, ISG15 transcription, and the generation of protective immune responses. J Immunol (2008) 180:7537-45. doi:10.4049/ jimmunol.180.11.7537

20. Neves BM, Silvestre R, Resende M, Ouaissi A, Cunha J, Tavares J, et al. Activation of phosphatidylinositol 3-kinase/Akt and impairment of nuclear factor-kappaB: molecular mechanisms behind the arrested maturation/ activation state of Leishmania infantum-infected dendritic cells. Am J Pathol (2010) 177:2898-911. doi:10.2353/ajpath.2010.100367

21. Resende M, Moreira D, Augusto J, Cunha J, Neves B, Cruz MT, et al. Leishmania-infected MHC class II high dendritic cells polarize CD4+ T cells toward a nonprotective T-bet+ IFN- $\gamma+$ IL-10+ phenotype. J Immunol (2013) 191:262-73. doi:10.4049/jimmunol.1203518

22. Marovich MA, McDowell MA, Thomas EK, Nutman TB. IL-12p70 production by Leishmania major-harboring human dendritic cells is a CD40/CD40 ligand-dependent process. JImmunol (2000) 164:5858-65. doi:10.4049/ jimmunol.164.11.5858

23. Brown JA, Greenwald RJ, Scott S, Schweitzer AN, Satoskar AR, Chung C, et al. $\mathrm{T}$ helper differentiation in resistant and susceptible B7-deficient mice infected with Leishmania major. Eur J Immunol (2002) 32:1764-72. doi:10.1002/1521-4141(200206)32:6<1764::AID-IMMU1764>3.0.CO;2-V

24. Carvalho AK, Carvalho K, Passero LF, Sousa MG, da Matta VL, Gomes CM, et al. Differential recruitment of dendritic cells subsets to lymph nodes correlates with a protective or permissive T-cell response during Leishmania (Viannia) braziliensis or Leishmania (Leishmania) amazonensis infection. Mediators Inflamm (2016) 2016:7068287. doi:10.1155/2016/7068287

25. Favali C, Tavares N, Clarêncio J, Barral A, Barral-Netto M, Brodskyn C. Leishmania amazonensis infection impairs differentiation and function of human dendritic cells. JLeukoc Biol (2007) 82:1401-6. doi:10.1189/ jlb.0307187

26. Soong L. Modulation of dendritic cell function by Leishmania parasites. J Immunol (2008) 180:4355-60. doi:10.4049/jimmunol.180.7.4355

27. Ishii N, Ndhlovu LC, Murata K, Sato T, Kamanaka M, Sugamura K. OX40 (CD134) and OX40 ligand interaction plays an adjuvant role during in vivo Th2 responses. Eur J Immunol (2003) 33:2372-81. doi:10.1002/eji.200324031

28. Akiba H, Miyahira Y, Atsuta M, Takeda K, Nohara C, Futagawa T, et al. Critical contribution of OX40 ligand to T helper cell type 2 differentiation in experimental leishmaniasis. J Exp Med (2000) 191:375-80. doi:10.1084/ jem.191.2.375

29. Tuladhar R, Oghumu S, Dong R, Peterson A, Sharpe AH, Satoskar AR. Ox40L-Ox40 pathway plays distinct roles in regulating Th2 responses but does not determine outcome of cutaneous leishmaniasis caused by Leishmania mexicana and Leishmania major. Exp Parasitol (2015) 148:49-55. doi:10.1016/j.exppara.2014.11.002
30. Liang SC, Greenwald RJ, Latchman YE, Rosas L, Satoskar A, Freeman GJ, et al. PD-L1 and PD-L2 have distinct roles in regulating host immunity to cutaneous leishmaniasis. Eur J Immunol (2006) 36:58-64. doi:10.1002/eji. 200535458

31. Winau F, Weber S, Sad S, de Diego J, Hoops SL, Breiden B, et al. Apoptotic vesicles crossprime $\mathrm{CD} 8 \mathrm{~T}$ cells and protect against tuberculosis. Immunity (2006) 24:105-17. doi:10.1016/j.immuni.2005.12.001

32. Gutiérrez-Kobeh L, de Oyarzabal E, Argueta J, Wilkins A, Salaiza N, Fernández E, et al. Inhibition of dendritic cell apoptosis by Leishmania mexicana amastigotes. Parasitol Res (2013) 112:1755-62. doi:10.1007/ s00436-013-3334-2

33. Bieber K, Autenrieth SE. Insights how monocytes and dendritic cells contribute and regulate immune defense against microbial pathogens. Immunobiology (2015) 220:215-26. doi:10.1016/j.imbio.2014.10.025

34. Xin L, Li K, Soong L. Down-regulation of dendritic cell signaling pathways by Leishmania amazonensis amastigotes. Mol Immunol (2008) 45:3371-82. doi:10.1016/j.molimm.2008.04.018

35. Contreras I, Estrada JA, Guak H, Martel C, Borjian A, Ralph B, et al. Impact of Leishmania mexicana infection on dendritic cell signaling and functions. PLoS Negl Trop Dis (2014) 8:e3202. doi:10.1371/journal.pntd.0003202

36. Brodskyn C, Beverley SM, Titus RG. Virulent or avirulent (dhfr-ts-) Leishmania major elicit predominantly a type-1 cytokine response by human cells in vitro. Clin Exp Immunol (2000) 119:299-304. doi:10.1046/j.1365-2249.2000.01122.x

37. Roy S, Mukhopadhyay D, Mukherjee S, Ghosh S, Kumar S, Sarkar K, et al. A defective oxidative burst and impaired antigen presentation are hallmarks of human visceral leishmaniasis. J Clin Immunol (2015) 35:56-67. doi:10.1007/ s10875-014-0115-3

38. Vieira ÉL, Keesen TS, Machado PR, Guimarães LH, Carvalho EM, Dutra WO, et al. Immunoregulatory profile of monocytes from cutaneous leishmaniasis patients and association with lesion size. Parasite Immunol (2013) 35:65-72. doi:10.1111/pim.12012

39. Revest M, Donaghy L, Cabillic F, Guiguen C, Gangneux JP. Comparison of the immunomodulatory effects of $L$. donovani and L. major excreted-secreted antigens, particulate and soluble extracts and viable parasites on human dendritic cells. Vaccine (2008) 26:6119-23. doi:10.1016/j.vaccine.2008.09.005

40. Colmenares M, Corbí AL, Turco SJ, Rivas L. The dendritic cell receptor DC-SIGN discriminates among species and life cycle forms of Leishmania. J Immunol (2004) 172:1186-90. doi:10.4049/jimmunol.172.2.1186

41. Markikou-Ouni W, Drini S, Bahi-Jaber N, Chenik M, Meddeb-Garnaoui A. Immunomodulatory effects of four Leishmania infantum potentially excreted/ secreted proteins on human dendritic cells differentiation and maturation. PLoS One (2015) 10:e0143063. doi:10.1371/journal.pone.0143063

42. Green PJ, Feizi T, Stoll MS, Thiel S, Prescott A, McConville MJ. Recognition of the major cell surface glycoconjugates of Leishmania parasites by the human serum mannan-binding protein. Mol Biochem Parasitol (1994) 66:319-28. doi:10.1016/0166-6851(94)90158-9

43. Liu D, Uzonna JE. The early interaction of Leishmania with macrophages and dendritic cells and its influence on the host immune response. Front Cell Infect Microbiol (2012) 2:83. doi:10.3389/fcimb.2012.00083

44. Lefèvre L, Lugo-Villarino G, Meunier E, Valentin A, Olagnier D, Authier H, et al. The C-type lectin receptors dectin-1, MR, and SIGNR3 contribute both positively and negatively to the macrophage response to Leishmania infantum. Immunity (2013) 38:1038-49. doi:10.1016/j.immuni.2013.04.010

45. Carvalho K, Vallejo M, Camargo Z, Puccia R. Use of recombinant gp43 isoforms expressed in Pichia pastoris for diagnosis of paracoccidioidomycosis. Clin Vaccine Immunol (2008) 15:622-9. doi:10.1128/CVI.00437-07

46. de Assis Souza M, de Castro MC, de Oliveira AP, de Almeida AF, de Almeida TM, Reis LC, et al. Cytokines and NO in American tegumentary leishmaniasis patients: profiles in active disease, after therapy and in self-healed individuals. Microb Pathog (2013) 57:27-32. doi:10.1016/j.micpath.2013.02.004

47. Oliveira WN, Ribeiro LE, Schrieffer A, Machado P, Carvalho EM, Bacellar $\mathrm{O}$. The role of inflammatory and anti-inflammatory cytokines in the pathogenesis of human tegumentary leishmaniasis. Cytokine (2014) 66:127-32. doi:10.1016/j.cyto.2013.12.016

48. Nateghi Rostami M, Seyyedan Jasbi E, Khamesipour A, Mohammadi AM. Tumour necrosis factor-alpha (TNF- $\alpha$ ) and its soluble receptor type 1 (sTNFR I) in human active and healed leishmaniases. Parasite Immunol (2016) 38:255-60. doi:10.1111/pim.12305 
49. Valdés-Reyes L, Argueta J, Morán J, Salaiza N, Hernández J, Berzunza M, et al. Leishmania mexicana: inhibition of camptothecin-induced apoptosis of monocyte-derived dendritic cells. Exp Parasitol (2009) 121:199-207. doi:10.1016/j.exppara.2008.10.020

50. Vázquez-López R, Argueta-Donohué J, Wilkins-Rodríguez A, EscalonaMontaño A, Aguirre-García M, Gutiérrez-Kobeh L. Leishmania mexicana amastigotes inhibit p38 and JNK and activate PI3K/AKT: role in the inhibition of apoptosis of dendritic cells. Parasite Immunol (2015) 37:579-89. doi:10.1111/pim. 12275

51. Li X, He Y. Caspase-2-dependent dendritic cell death, maturation, and priming of T cells in response to Brucella abortus infection. PLoS One (2012) 7:e43512. doi:10.1371/journal.pone.0043512
Conflict of Interest Statement: The authors declare that the research was conducted in the absence of any commercial or financial relationships that could be construed as a potential conflict of interest.

Copyright (C) 2016 Falcão, Jaramillo, Ferreira, Bernardes, Santana and Favali. This is an open-access article distributed under the terms of the Creative Commons Attribution License (CC BY). The use, distribution or reproduction in other forums is permitted, provided the original author(s) or licensor are credited and that the original publication in this journal is cited, in accordance with accepted academic practice. No use, distribution or reproduction is permitted which does not comply with these terms. 